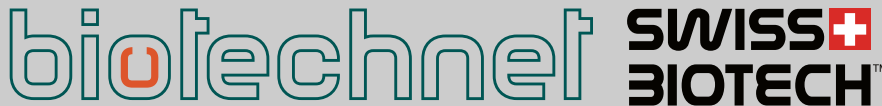

biotechnet Switzerland

\section{ZHAW Waedenswil: A new Approach in the Fight against Cancer}

\section{Markus Rimann*}

${ }^{*}$ Correspondence: Dr M. Rimann, Group Leader 3D Tissues and Biofabrication, ICBT , ZHAW Zurich University of Applied Sciences, Waedenswil, E-Mail: markus. rimann@zhaw.ch

Abstract: A happy coincidence brought Dr Markus Rimann from ZHAW Waedenswil together with Dr Andreas Meyer from the start-up FGen and PD Dr Emanuela Felley-Bosco, Molecular Oncologist at Zurich University Hospital, to develop a technology platform for the manufacture and high throughput analysis of single mesothelioma spheroids. Armin Picenoni, former student in Chemistry for the Life Sciences at ZHAW, confirmed everything in writing his Master Thesis on this Innosuisse project.

Cancer cells are insidious. While normal cells obey signals that tell them when they have reached their limit and will cause damage if they grow any further, cancer cells suspend the operation of the normal signalling system. They don't stop growing and dividing, and escape the programmed cell death known as apoptosis. The processes and mechanisms behind this behaviour and the process of tumor formation are very complex, multi-layered, and still largely unresolved.

\section{The Silent Killer}

CEO Dr Andreas Meyer founded the start-up company FGen $\mathrm{GmbH}$ with three colleagues in Basel in 2011. The idea behind the spin-off of the Department of Biosystems Science and Engineering at ETH Zurich was to combine biological engineering and high throughput screening so as to design microbial strains that would permit the efficient and cost-competitive bio-production of chemicals and proteins. In order to enlarge the scope of FGen's proprietary screening platform and access new markets, Dr Meyer wanted to introduce mammalian cell analysis into the portfolio. This led him to contact Dr Markus Rimann, Group Leader 3D Tissues and Biofabrication at ZHAW Waedenswil. He is interested in creating predictive and reproducible cell culture systems for drug development in order to save costs, reduce the need for animal experiments and shorten long development times. They had the idea of providing cells with different mechanical microenvironments since cells sense their environment through mechano-transducers. Cells depend on external forces acting on transcription via signal cascades to stop or advance cell growth. The mechanical microenvironment of the cells can be modified in specific ways using hydrogels. Recently, it has been recognized that cancer cells behave differently when cultured in hydrogels with different mechanical properties. For this reason, Dr Meyer, who earned his $\mathrm{PhD}$ in biotechnology at ETH Zurich, wanted to introduce cancer cells into hydrogels employing FGen's technology. Hydrogels would make it possible not only to cultivate cells in $3 \mathrm{D}$, like in the human body, but also to promote the growth of specific cell populations that could be particularly resistant to can- cer therapy by changing the mechanical properties. Thanks to this cell amplification, it would be possible in the future to specifically develop drugs against these particular cells.

PD Dr Emanuela Felley-Bosco was asked to join the team as a third research partner. She is a molecular oncologist at Zurich University Hospital and an expert in malignant pleural mesothelioma (MPM), a cancer associated with exposure to asbestos fibers. Although the commercial use of asbestos was banned in the early nineties, the incidence of MPM will continue to rise in Western Europe over the next decade because of the latency period of up to 40 years. Innosuisse - formerly the CTI - was ready to support the research and development work of the winwin partnership for two years.

As part of the project, FGen used its special Nanoliter Reactor (NLR) Technology to introduce mesothelioma cells - cancer triggered by asbestos - by high throughput into alginate hydrogels and use them for drug screening. Alginate is an old acquaintance: it's an acidic polysaccharide formed on the cell walls of brown algae (Phaeophyceae), which gives them their typical flexibility. In medicine it is used in medical dressings as calcium alginate, where it forms a gel and keeps wounds fresh. In the project three different alginates with different guluronate to mannuronate $(\mathrm{G} / \mathrm{M})$ ratios and viscosities were used to modify hydrogel stiffness.

\section{Working steadily toward the Goal}

To achieve the objective of developing a high throughput screening technology for the phenotypic analysis of mammalian cell libraries, Armin Picenoni tried to manually encapsulate mammalian cells in hydrogel droplets in the microliter volume range (Fig. 1). The crosslinked beads served as pre-study objects for further translation to the fully automated NLR technology. In his study, Picenoni evaluated different alginate hydrogel sources with respect to the cultivation of a mesothelioma cell line, which would serve as model for setting up the NLR technology with mammalian cells at the beginning of the project. Altering the stiffness of the hydrogel, the researchers intended to mimic the in vivo microenvironment of the tumour cells so they could cultivate and analyse the cell line. The final goal is to use the characterized hydrogels to encapsulate cells obtained from biopsies from patients with mesothelioma in order to enrich and select specific subpopulations of cells, such as cancer stem cells or primary cancer cells.

This ultimately makes it possible to generate clonal patientderived cell lines which can, for example, be used in drug testing or as cancer cell models. "Based on the results of this project, we expect it will be possible to develop other high throughput screening protocols for analyzing mammalian cell libraries", explains Armin Picenoni. "The NLR platform is capable of offering a unique technology for the high throughput phenotypic analysis of 3D cultured mammalian cells that has not been possible up to now using current methods." That helps to find cells with the desired properties: The NLR technology permits phenotypic analysis of cell libraries at rates of more than 1 million clones per day. That in turn, makes it possible to screen large libraries generated by classic non-GMO methods or combinatorial design 


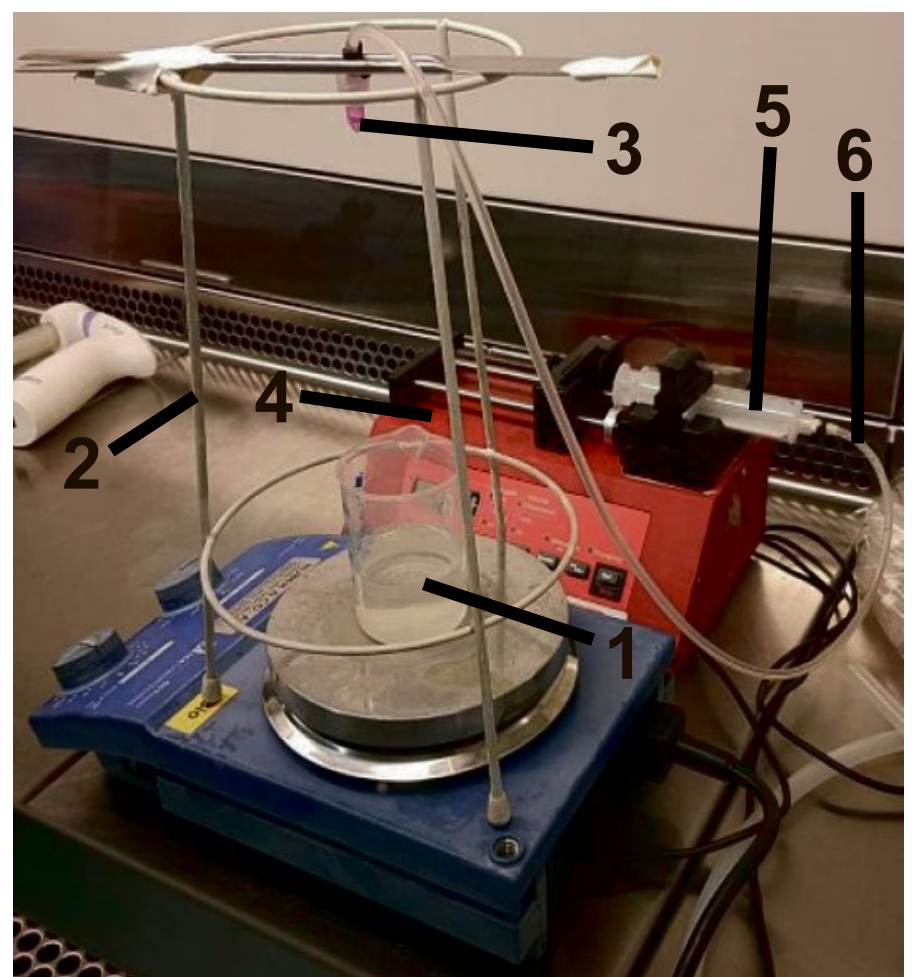

Fig. 1. Equipment for manual cell encapsulation into Ca-alginate beads. Cell-containing alginate is dropped into the calcium bath for crosslinking: 1) $100 \mathrm{ml}$ beaker containing a calcium chloride bath to crosslink the alginate droplets; 2) Holder for needle tip; 3) Needle tip to produce alginate droplets; 4) Syringe pump; 5) $20 \mathrm{ml}$ syringe containing alginate solution; 6) Tube connecting the alginate-containing syringe with the needle tip. (Source: Master's thesis Armin Picenoni)

for example, and increases the probability of finding an improved candidate.

\section{Stay 'on the ball'}

Throughout the project, Armin Picenoni persistently pursued the interesting trails, analyzing growth, histology and structure, modulating the stiffness for cells with special properties, conducting rheological studies to analyze the viscosity of the alginates, substituting barium ions for calcium ions, so as to achieve harder alginate beads, and performing measurements with the atomic force microscope to determine the E-modulus (stiffness) (Table 1). Dr Markus Rimann remembers: "He developed an amazing zeal, always with the goal in mind!" For cell encapsulation, a mesothelioma cell line, alginate beads of $1 \mathrm{~mm}$ to $1.6 \mathrm{~mm}$ in diameter were produced. "We performed the encapsulation by simple capillary jet instability and then crosslinked the capsules ionically in a $\mathrm{CaCl}_{2}$ solution," recalls Armin Picenoni. "We determined the E-modulus of these cured, $2 \%$ alginates by rheometry and atomic force microscopic indentation measurements at $37^{\circ} \mathrm{C}$." The cell spheroids grown possessed an average diameter of $40-55 \mu \mathrm{m}$ and were often oval in shape after fourteen days of culture (Fig. 2). Hematoxylin and eosin staining revealed densely packed cell spheroids in all alginate capsules. "Calcium alginates with a smaller E-modulus led to larger but fewer cell spheroids compared to stiffer alginate beads", the scientist concludes, who is grateful to his mentor Markus Rimann to have learned a great deal thanks to this project work. "The results give a good first impression of the cells' behaviour in calcium alginates with different E-moduli. The methods we have established pave the way for further experiments in the direction of mechanical cell manipulation and integration of primary cancer cell material."

\section{Faster and more Accurate}

The analysis of large cell libraries is an important process in pharmaceutical discovery and $\mathrm{R} \& \mathrm{D}$, and the technology developed in this project will find application in various areas. FGen will use the NLR platform to construct patient-derived primary cell lines (Fig. 3). Such cell lines are important tools in pharmaceutical assays such as the evaluation of drug efficacy. Since these tests can be performed in modified alginates that permit $3 \mathrm{D}$ cell growth while mimicking the in vivo microenvironment, improved predictability of drug potency may be expected. Now that the feasibility of the NLR technology for the analysis of mammalian cell libraries has been proven, FGen plans to implement other assay formats such as screening whole genome CRISPR libraries for target identification or screening B-cells secreting antibodies toward a specific target. For the time being, FGen is mainly active in the field of industrial biotechnology and performs contract research for the engineering and screening of microbial strains. Also being able to offer services in the area of mammalian cell assay
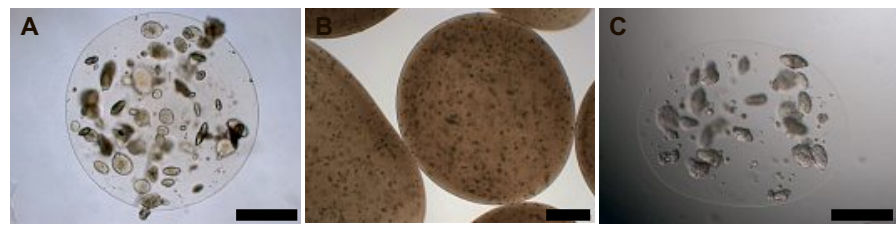

Fig. 2. Mesothelioma cells were grown for 14 days in different alginate beads. Cells grown in A (A), B (B) and C alginate (C). Scale bar $=500 \mu \mathrm{m}$. (Source: ZHAW)

Table 1. Chemical and mechanical properties of different alginates in $2 \%$ concentration polymerized in $50 \mathrm{mM} \mathrm{CaCl}_{2}$ (G/M ratio: guluronate / mannuronate ratio). (Source: ZHAW)

\begin{tabular}{|l|l|l|l|}
\hline Alginate & G/M ratio & \multicolumn{2}{|l|}{ E-modulus [kPa] } \\
\hline & & Rheometry & $\begin{array}{l}\text { Indentation } \\
\text { (AFM) }\end{array}$ \\
\hline Cimalgin 500 (A) & 1.4 & $4.7 \pm 0.6$ & $6.9 \pm 1.1$ \\
\hline $\begin{array}{l}\text { Cimalgin 80/400 } \\
\text { (B) }\end{array}$ & $1.1-1.3$ & $10.3 \pm 1.7$ & $8.7 \pm 2.0$ \\
\hline Sigma (C) & $0.5-0.6$ & $1.7 \pm 0.1$ & $2.4 \pm 2.1$ \\
\hline
\end{tabular}
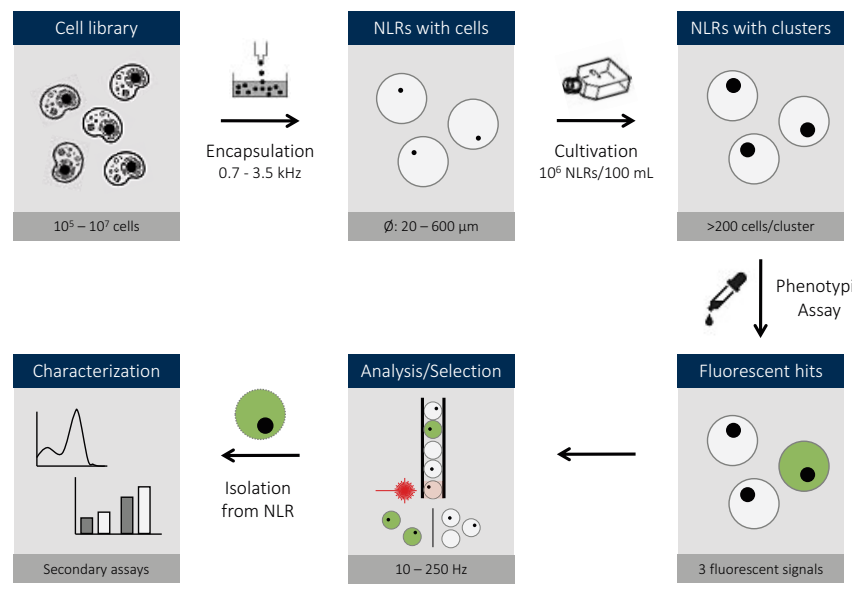

Fig. 3. NLR technology scheme. (Source: FGen) 
development and screening, for which this project delivers the proof-of-concept, is expected to double the current turnover of FGen within the next few years and enables FGen to continue its organic growth strategy.

FGen's research partners ZHAW and USZ are also winners because the project broke ground in a new and innovative field using an established material in the form of alginate. "Combining established technologies, such as 3D cell cultures with alginates as versatile hydrogels in a high throughput-compatible manner drives cancer and industrial research! It is crucial to bring together expertise, experience, research and development in a partnership where both academia and the market are driven by the irrepressible will to open up completely new horizons and to bring successful products to the global market!"

\section{Acknowledgements}

We gratefully thank the innovative promotion agency (Innosuisse, Switzerland, Project No.: 18364.2) for financial support of the project and technical guidance.
For more information, please contact:

Armin Picenoni, MSc, E-mail: a.picenoni@bluewin.ch Dr Markus Rimann, ZHAW ICBT Waedenswil

Tel.: +41589345512

E-mail: markus.rimann@zhaw.ch

URL: www.zhaw.ch/icbt/3d-tissues-and-biofabrication/

Dr Emanuela Felley-Bosco, University Hospital Zurich, Zurich

Tel.: +41 442552771

E-mail: Emanuela.Felley-Bosco@usz.ch

URL: http://www.zkf.uzh.ch/de/ResearchGroups/Stahel.html

Dr Andreas Meyer, CEO FGen GmbH, Basel

Tel.: +41616332920

URL: http://www.fgen.ch 